\title{
Passive Synthetic Aperture Radar Imaging with Single Frequency Sources of Opportunity
}

\author{
Can Evren Yarman ${ }^{1}$, Ling Wang ${ }^{2}$, Birsen Yazıci ${ }^{3}$ \\ ${ }^{1}$ Houston Technology Center, WesternGeco Schlumberger, Houston, TX 77042 USA \\ ${ }^{2}$ College of Information Science and Technology,Nanjing University of Aeronautics and Astronautics,Nanjing,210016 China \\ ${ }^{3}$ Department of Electrical, Computer and System Engineering, Rensselaer Polytechnic Institute, Troy, NY 12180 USA \\ E-mail: yazici@ecse.rpi.edu
}

\begin{abstract}
In this paper we consider passive airborne receivers that use backscattered signals from sources of opportunity transmitting fixed-frequency waveforms. Due to its combined passive synthetic aperture and the fixed-frequency nature of the transmitted waveforms, we refer to the system under consideration as Doppler Synthetic Aperture Hitchhiker (DSAH). We present a novel image formation method for DSAH. Our method first correlates the windowed signal obtained from one receiver with the windowed, filtered, scaled and translated version of the received signal from another receiver. This processing removes the transmitter related variables from the phase of the Fourier integral operator that maps the radiance of the scene to the correlated signal. We next use the microlocal analysis to reconstruct the scene radiance by the weightedbackprojection of the correlated signal. This imaging algorithm can put the visible edges of the scene radiance at the correct location, and under appropriate conditions, with correct strength. Additionally, it is an analytic reconstruction technique which can be made computationally efficient. We show that the resolution of the image is directly related to the length of the support of the windowing function and the frequency of the transmitted waveform. The image reconstruction method is applicable with both cooperative and non-cooperative sources of opportunity using one or more airborne receivers.
\end{abstract}

We present numerical simulations to demonstrate the performance of the image reconstruction method and to verify the theoretical results.

\section{INTRODUCTION}

In recent years, there has been a growing interest in passive radar applications using sources of opportunity [1]-[3]. This research effort is motivated by the growing availability of transmitters of opportunity, such as radio, television and cell phone stations, particularly in urban areas, as well as relatively low cost and rapid deployment of passive receivers.

While many of the passive radar applications are focused on the detection of airborne targets with ground based receivers recently, a number of methods for passive synthetic aperture radar has been developed. In [4], we reported a novel passive synthetic aperture imaging method that is based on the spatio-temporal correlation of the received signal and the filtered-backprojection technique. This method does not require receivers with high directivity or a priori knowledge about the transmitter locations and transmitted waveforms. The

${ }^{1}$ This work is supported by the Air Force Office of Scientific Research(AFOSR) under the agreement FA9550-07-1-0363 and by the National Science Foundation (NSF) under Grant No. CCF-08030672. resolution analysis of the method shows that it is suitable for high-range-resolution waveforms, such as wideband pulses. However, most of the transmitters of opportunity, such as radio and TV stations, transmit single frequency waveforms. In this paper, we present a new passive synthetic aperture imaging method using sources of opportunity transmitting fixedfrequency waveforms. These waveforms are also referred to as high-Doppler-resolution or continuous-wave (CW) waveforms. Thus, we refer to the resulting method as the Doppler Synthetic Aperture Hitchhiker (DSAH) imaging method.

Our passive imaging method has the following advantages: (1) it does not require receivers with high directivity; (2) it can be used in the presence of both cooperative and noncooperative sources of opportunity; (3) it can be used with stationary and/or mobile sources of opportunity; (4) it can be used with one or more airborne receivers; (5) it can be used under non-ideal imaging scenarios such as arbitrary flight trajectories and non-flat topography; (6) it has the desirable property of preserving the visible edges of the scene radiance in the reconstructed image. Additionally, it is an analytic image formation method that can be made computationally efficient.

The organization of our paper is as follows: In Section II, we first introduce the model of the received signal. Then we develop he forward model for DSAH and analyze the leading-order contributions to the windowed, filtered-scaledand-correlated measurements. In Section III, we develop a weighted-backprojection type image formation method for DSAH and analyze the underlying geometry and resolution of DSAH image formation. In Section IV, we present numerical simulations to verify the theoretical results and to demonstrate the performance of the DSAH image formation method. Finally, in Section V we conclude our discussion.

\section{DSAH MEASUREMENT MODEL}

\section{A. Model for the received signal}

We use the following notational conventions throughout the paper. The bold Roman, bold italic and Roman lowercase letters are used to denote variables in $\mathbb{R}^{3}, \mathbb{R}^{2}$ and $\mathbb{R}$, respectively, i.e., $\mathbf{z}=(\boldsymbol{z}, z) \in \mathbb{R}^{3}$, with $\boldsymbol{z} \in \mathbb{R}^{2}$ and $z \in \mathbb{R}$. The calligraphic letters $(\mathcal{F}, \mathcal{K}$ etc. $)$ are used to denote operators.

We make the assumption that the earth's surface is located at a position given by $\mathbf{z}=(\boldsymbol{z}, \boldsymbol{\psi}(\boldsymbol{z})) \in \mathbb{R}^{3}$, where $\boldsymbol{z} \in \mathbb{R}^{2}$ and 


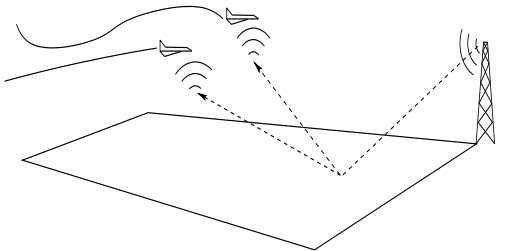

Fig. 1: An illustration of the DSAH imaging geometry.

$\psi: \mathbb{R}^{2} \rightarrow \mathbb{R}$ is a known function for the ground topography. Furthermore, we assume that the scattering takes place in a thin region near the surface.

Under these assumptions, for a fixed-frequency waveform at frequency $\omega_{0}$, given a pair of transmitter and receiver antennas located at $\mathbf{T}$ and $\mathbf{R}$ respectively, we model the received signal by [5]

$$
\begin{aligned}
& f(t, \mathbf{R}, \mathbf{T}) \approx \int \frac{\mathrm{e}^{-\mathrm{i} \omega_{0}\left(t-(|\mathbf{R}-\mathbf{z}|+|\mathbf{z}-\mathbf{T}|) / c_{0}\right)}}{(4 \pi)^{2}|\mathbf{R}-\mathbf{z}||\mathbf{z}-\mathbf{T}|} \omega_{0}^{2} \\
& \times J_{\mathrm{tr}}\left(\omega_{0}, \widehat{\mathbf{z}-\mathbf{T}}, \mathbf{T}\right) J_{\mathrm{rc}}\left(\omega_{0}, \widehat{\mathbf{z}-\mathbf{R}}, \mathbf{R}\right) \rho(\boldsymbol{z}) d \boldsymbol{z}
\end{aligned}
$$

where $t$ denotes time, $c_{0}$ denotes the speed of light in freespace, $\rho(\boldsymbol{z})$ is the reflectivity function, and $J_{t r}$ and $J_{r c}$ are the transmitter and receiver antenna beam patterns, respectively. $\widehat{\mathbf{z}}=\mathbf{z} /|\mathbf{z}|$ denotes the unit vector in the direction of $\mathbf{z} \in \mathbb{R}^{3}$. For the rest of the paper, unless otherwise stated, we use $\mathbf{z}=$ $\mathbf{z}(\boldsymbol{z})=(\boldsymbol{z}, \boldsymbol{\psi}(\boldsymbol{z}))$.

We assume that there is a single, stationary transmitter of opportunity illuminating the scene. Let $\mathbf{T} \in \mathbb{R}^{3}$ denote the location of the transmitter and let there be $N$ airborne receivers, each traversing a smooth trajectory $\gamma_{i}(t), i=1, \cdots, N$ as shown in Figure 1. We define

$$
s_{i}(t)=f\left(t, \gamma_{i}(t), \mathbf{T}\right)
$$

as the received signal by the $i$ th receiver, $i=1,, \cdots, N$.

Note that our imaging method is applicable to both mobile and stationary transmitter and also applicable to multiple transmitters and multiple transmit frequencies.

\section{B. Windowed, filtered-scaled-and-translated correlation of re- ceived signals}

For a given point of interest $\boldsymbol{z}_{0}$ on the ground topography and a fixed $\tau^{\prime} \in \mathbb{R}$, we define the windowed, filtered-scaledand-translated correlation of the received signals $s_{i}$ and $s_{j}$ by

$$
c_{i j}^{z_{0}}\left(\tau^{\prime}, \tau, \mu\right)=\int \frac{s_{i}\left(t+\tau^{\prime}\right) s_{j}^{*}(\mu t+\tau)}{B_{i j}\left(\boldsymbol{z}_{0}, t, \tau^{\prime}, \tau, \mu\right)} \phi(t)|t| d t,
$$

for some $\tau, \tau^{\prime} \in \mathbb{R}$ and $\mu \in \mathbb{R}^{+}, i, j=1, \cdots, N$, where $\phi(t)$ is a smooth compactly supported temporal windowing function centered at $t=0$; and $B_{i j}$ is a filter to be determined later; and ${ }^{*}$ denotes the complex conjugation. To simplify our notation, we drop the superscripts $\boldsymbol{z}_{0}$ from $c_{i j}^{z_{0}}\left(\tau^{\prime}, \tau, \mu\right)$ for the rest of our paper. Note that for a single receiver, we only have $c_{i j}=c_{11}$.
We make the incoherent-field approximation [6] by assuming that $\rho$ and $J_{\operatorname{tr}}$ satisfy the following equalities:

$$
\begin{aligned}
C_{\rho}\left(\boldsymbol{z}, \boldsymbol{z}^{\prime}\right) & =R_{\rho}(\boldsymbol{z}) \delta\left(\boldsymbol{z}-\boldsymbol{z}^{\prime}\right) . \\
C_{J_{\mathrm{tr}}}\left(\omega_{0}, \boldsymbol{z}, \boldsymbol{z}^{\prime}, \mathbf{T}\right) & =R_{T}\left(\omega_{0}, \boldsymbol{z}, \boldsymbol{z}^{\prime}, \mathbf{T}\right) \delta\left(\boldsymbol{z}-\boldsymbol{z}^{\prime}\right) .
\end{aligned}
$$

where $C_{\rho}$ and $C_{J_{\mathrm{tr}}}$ denote the correlation function of $\rho$ and $J_{\mathrm{tr}}$, respectively. Note that $R_{\rho}$ is the average power of the electromagnetic radiation emitted by the scene at location $\boldsymbol{z}$, and $R_{T}$ is the average power of the electromagnetic radiation emitted by the transmitter at location $\mathbf{T}$ that is incident on the target surface at $\boldsymbol{z}$. In this regard, $R_{\rho}$ is referred to as the scene radiance and $R_{T}$ is referred to as the transmitter irradiance [6].

Substituting $s_{i}$ and $s_{j}$ into $c_{i j}$, under the assumption that $\rho$ and $J_{\text {tr }}$ are statistically independent, and using (4) and (5), we express the expectation of $c_{i j}$ as follows:

$$
\begin{aligned}
& E\left[c_{i j}\left(\tau^{\prime}, \tau, \mu\right)\right] \\
& =\frac{\omega_{0}^{4}}{(4 \pi)^{4}} \int \mathrm{e}^{-\mathrm{i} \omega_{0}\left(t+\tau^{\prime}-\left(\left|\boldsymbol{\gamma}_{i}\left(t+\tau^{\prime}\right)-\mathbf{z}\right|+|\mathbf{T}-\mathbf{z}|\right) / c_{0}\right)} \\
& \times \mathrm{e}^{\mathrm{i} \omega_{0}\left(\mu t+\tau-\left(\left|\boldsymbol{\gamma}_{j}(\mu t+\tau)-\mathbf{z}^{\prime}\right|+\left|\mathbf{T}-\mathbf{z}^{\prime}\right|\right) / c_{0}\right)} \\
& \times \frac{R_{T}\left(\omega_{0}, \boldsymbol{z}, \boldsymbol{z}^{\prime}, \mathbf{T}\right) A_{R_{i j}}\left(\omega_{0}, \boldsymbol{z}, \boldsymbol{z}^{\prime}, t, \tau^{\prime}, \tau, \mu\right)}{G_{i j}\left(\mathbf{z}, \mathbf{z}^{\prime}, t, \tau^{\prime}, \tau, \mu\right) B_{i j}\left(\boldsymbol{z}_{0}, t, \tau^{\prime}, \tau, \mu\right)} \\
& \times R_{\rho}(\boldsymbol{z}) \delta\left(\boldsymbol{z}-\boldsymbol{z}^{\prime}\right) d \boldsymbol{z} d \boldsymbol{z}^{\prime} \phi(t)|t| d t
\end{aligned}
$$

where $A_{R_{i j}}$ is the product of the receiver antenna beam patterns,

$$
\begin{aligned}
& A_{R_{i j}}\left(\omega_{0}, \boldsymbol{z}, \boldsymbol{z}^{\prime}, t, \tau^{\prime}, \tau, \mu\right) \\
& =J_{\mathrm{rc}}\left(\omega_{0}, \mathbf{z}-\widehat{\left.\boldsymbol{\gamma}_{i}\left(t+\tau^{\prime}\right), \gamma_{i}\left(t+\tau^{\prime}\right)\right)}\right. \\
& \left.\times J_{\mathrm{rc}}^{*}\left(\omega_{0}, \mathbf{z}^{\prime}-\widehat{\boldsymbol{\gamma}_{j}(\mu t}+\tau\right), \gamma_{j}(\mu t+\tau)\right)
\end{aligned}
$$

and $G_{i j}$ is the product of the geometric spreading factors,

$$
\begin{aligned}
& G_{i j}\left(\mathbf{z}, \mathbf{z}^{\prime}, t, \tau^{\prime}, \tau, \mu\right)=|\mathbf{T}-\mathbf{z}|\left|\mathbf{T}-\mathbf{z}^{\prime}\right| \\
& \quad \times\left|\gamma_{i}\left(t+\tau^{\prime}\right)-\mathbf{z}\right|\left|\gamma_{j}(\mu t+\tau)-\mathbf{z}^{\prime}\right| .
\end{aligned}
$$

Note that for non-cooperative sources of opportunity, $\mathbf{T}$ and thus $|\mathbf{T}-\mathbf{z}|\left|\mathbf{T}-\mathbf{z}^{\prime}\right|$, are unknown.

Now using the Taylor series expansion of $\left|\gamma_{i}\left(t+\tau^{\prime}\right)-\mathbf{z}\right|$ and $\left|\gamma_{j}(\mu t+\tau)-\mathbf{z}^{\prime}\right|$ at $t=0$, substituting the approximations back into (6), and performing the $\boldsymbol{z}^{\prime}$ integration, we have

$$
\begin{aligned}
& E\left[c_{i j}\left(\tau^{\prime}, \tau, \mu\right)\right] \approx \mathcal{F}_{i j}\left[R_{\rho}\right](\tau, \mu) \\
& =\int \mathrm{e}^{-\mathrm{i} \varphi_{i j}\left(t, \boldsymbol{z}, \tau^{\prime}, \tau, \mu\right)} \frac{A_{i j}\left(\boldsymbol{z}, t, \tau^{\prime}, \tau, \mu\right)}{B_{i j}\left(\boldsymbol{z}_{0}, t, \tau^{\prime}, \tau, \mu\right)} R_{\rho}(\boldsymbol{z}) d \boldsymbol{z}|t| d t(10)
\end{aligned}
$$

where

$$
\begin{aligned}
& \varphi_{i j}\left(t, \boldsymbol{z}, \tau^{\prime}, \tau, \mu\right)= \\
& \omega_{0} t\left[1-\left(\boldsymbol{\gamma}_{j} \widehat{(\tau)-\mathbf{z}}\right) \cdot \dot{\gamma}_{j}(\tau) / c_{0}\right]\left[S_{i j}\left(\tau^{\prime}, \tau, \mathbf{z}\right)-\mu\right]
\end{aligned}
$$

with

$$
S_{i j}\left(\tau^{\prime}, \tau, \mathbf{z}\right)=\frac{1-\left(\gamma_{i} \widehat{\left(\tau^{\prime}\right)}-\mathbf{z}\right) \cdot \dot{\gamma}_{i}\left(\tau^{\prime}\right) / c_{0}}{1-\left(\widehat{\gamma_{j}(\tau)}-\mathbf{z}\right) \cdot \dot{\gamma}_{j}(\tau) / c_{0}}
$$


and

$$
\begin{aligned}
& A_{i j}\left(\boldsymbol{z}, t, \tau^{\prime}, \tau, \mu\right)=\frac{\tilde{R}_{T}\left(\omega_{0}, \boldsymbol{z}\right) A_{R_{i j}}\left(\omega_{0}, \boldsymbol{z}, \boldsymbol{z}, t, \tau^{\prime}, \tau, \mu\right)}{G_{i j}\left(\mathbf{z}, \mathbf{z}, t, \tau^{\prime}, \tau, \mu\right)} \\
& \times \frac{\omega_{0}^{4} \phi(t)}{(4 \pi)^{4}} \mathrm{e}^{-\mathrm{i} \omega_{0}\left(\tau^{\prime}-\tau-\left(\left|\boldsymbol{\gamma}_{i}\left(\tau^{\prime}\right)-\mathbf{z}\right|-\left|\boldsymbol{\gamma}_{j}(\tau)-\mathbf{z}\right|\right) / c_{0}\right)}
\end{aligned}
$$

with $\tilde{R}_{T}\left(\omega_{0}, \boldsymbol{z}\right)=R_{T}\left(\omega_{0}, \boldsymbol{z}, \boldsymbol{z}, \mathbf{T}\right)$. We refer to $S_{i j}\left(\tau^{\prime}, \tau, \mathbf{z}\right)$ as the Doppler-hitchiker-scale-factor, and $\varphi_{i j}$ and $A_{i j} / B_{i j}$ as the phase and amplitude terms of the linear operator $\mathcal{F}_{i j}$.

For cooperative sources of opportunity, $\tilde{R}_{T}\left(\omega_{0}, \boldsymbol{z}\right)$ in (13) is replaced with $J_{\operatorname{tr}}\left(\omega_{0}, \widehat{\mathbf{z}-\mathbf{T}}, \mathbf{T}\right) J_{\operatorname{tr}}^{*}\left(\omega_{0}, \widehat{\mathbf{z}^{\prime}-\mathbf{T}}, \mathbf{T}\right)$.

Note that the filtered-scaled-and-translated correlation of the received signal removes all transmitter related terms from the phase of the operator $\mathcal{F}_{i j}$.

We assume that for some $m_{A}, A_{i j} / B_{i j}$ satisfies the following inequality:

$$
\begin{aligned}
& \sup _{(t, \mu, \tau, \boldsymbol{z}) \in \mathcal{U}}\left|\partial_{t}^{\alpha_{t}} \partial_{\mu}^{\alpha_{\mu}} \partial_{\tau}^{\beta} \partial_{z_{1}}^{\epsilon_{1}} \partial_{z_{2}}^{\epsilon_{2}} \frac{A_{i j}\left(\boldsymbol{z}, t, \tau^{\prime}, \tau, \mu\right)}{B_{i j}\left(\boldsymbol{z}_{0}, t, \tau^{\prime}, \tau, \mu\right)}\right| t|| \\
& \leq C_{A}\left(1+t^{2}\right)^{\left(m_{A}-\left|\alpha_{t}\right|\right) / 2}
\end{aligned}
$$

where $\mathcal{U}$ is any compact subset of $\mathbb{R}^{+} \times \mathbb{R}^{+} \times \mathbb{R} \times \mathbb{R} \times$ $\mathbb{R}^{2}$, and the constant $C_{A}$ depends on $\mathcal{U}, \alpha_{t, \mu}, \beta, \epsilon_{1,2}$. This assumption is needed in order to make various stationary phase calculations hold. Under the assumption (14), (10) defines $\mathcal{F}$ as a Fourier integral operator whose leading order contributions come from those points lying in the intersection of the illuminated surface $(\boldsymbol{z}, \boldsymbol{\psi}(\boldsymbol{z}))$ and points that have the same Doppler-hitchhiker-scale-factor, i.e., $\left\{\mathbf{z} \in \mathbb{R}^{3}: S_{i j}\left(\tau^{\prime}, \tau, \mathbf{z}\right)=\right.$ $\mu\}$. We denote the curves formed by this intersection by

$$
F_{i j}\left(\tau^{\prime}, \tau, \mu\right)=\left\{\boldsymbol{z}: S_{i j}\left(\tau^{\prime}, \tau, \mathbf{z}=(\boldsymbol{z}, \psi(\boldsymbol{z}))\right)=\mu\right\} .
$$

When the speed of the receivers is much slower than the speed of light $c_{0}, S_{i j}\left(\tau^{\prime}, \tau, \mathbf{z}\right)=\mu$ defines a hitchhiker Doppler [4] for a fixed frequency $\omega_{0}$. In this regard, we refer to $F_{i j}\left(\tau^{\prime}, \tau, \mu\right)$ as the DSAH iso-Doppler contour. Fig.2 shows the DSAH iso-Doppler contours for two receivers traversing a circular trajectory over a flat topography.

\section{IMAGE FORMATION}

We form an image of the scene radiance by the superposition of the weighted and backprojected data, $E\left[c_{i j}\left(\tau^{\prime}, \tau, \mu\right)\right]$ as follows:

$$
\tilde{R}_{\rho}(\boldsymbol{z})=\sum_{i j} \int \mathcal{K}_{i j}\left[E\left[c_{i j}\right]\right]\left(\boldsymbol{z}, \tau^{\prime}\right) d \tau^{\prime}
$$

where

$$
\begin{aligned}
& \mathcal{K}_{i j}\left[E\left[c_{i j}\right]\right]\left(\boldsymbol{z}, \tau^{\prime}\right)=\int \mathrm{e}^{\mathrm{i} \varphi_{i j}\left(t, \boldsymbol{z}, \tau^{\prime}, \tau, \mu\right)} Q_{i j}\left(\boldsymbol{z}, \tau^{\prime}, \tau, \mu\right) \\
& \times E\left[c_{i j}\left(\tau^{\prime}, \tau, \mu\right)\right] d t d \tau d \mu .
\end{aligned}
$$

We refer to $\mathcal{K}_{i j}$ as the weighted-backprojection operator with respect to the $i$ th and $j$ th receivers with weight $Q_{i j}$ to be determined below.

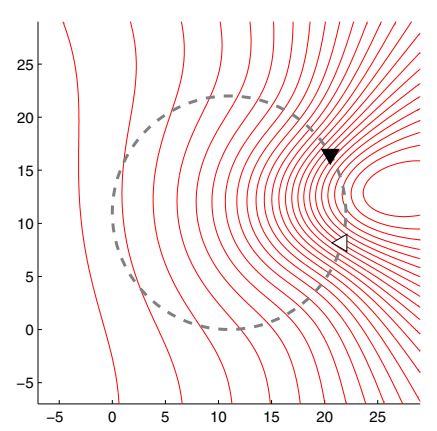

Fig. 2: Iso-Doppler contours $F_{i j}\left(\tau^{\prime}, \tau, \mu\right)$ for the Dopplerhitchhiker-scale-factor $S_{12}(\pi / 6,-\pi / 12, \mathbf{z})$. with $\mu$ sampling over $[1-1.5 \mathrm{e}-6,1+1.5 \mathrm{e}-6]$. Two receivers are traversing a circular flight trajectory (dashed line) at the speed of $261 \mathrm{~m} / \mathrm{s}$ over a flat topography. The white and black triangles denote the positions of the two receivers.

We rewrite $\tilde{R}_{\rho}$ as

$$
\tilde{R}_{\rho}(\boldsymbol{z})=\sum_{i j} \mathcal{K}_{i j} \mathcal{F}_{i j}\left[R_{\rho}\right](\boldsymbol{z})=\int L\left(\boldsymbol{z}, \boldsymbol{z}^{\prime}\right) R_{\rho}\left(\boldsymbol{z}^{\prime}\right) d \boldsymbol{z}^{\prime}
$$

where $L\left(\boldsymbol{z}, \boldsymbol{z}^{\prime}\right)$ is the point spread function (PSF) of the imaging operator given by

$$
L\left(\boldsymbol{z}, \boldsymbol{z}^{\prime}\right)=\sum_{i j} \int L_{i j}\left(\boldsymbol{z}, \boldsymbol{z}^{\prime}, \tau^{\prime}\right) d \tau^{\prime}
$$

and

$$
\begin{aligned}
& L_{i j}\left(\boldsymbol{z}, \boldsymbol{z}^{\prime}, \tau^{\prime}\right)=\int \mathrm{e}^{\mathrm{i}\left[\varphi_{i j}\left(t, \boldsymbol{z}, \tau^{\prime}, \tau, \mu\right)-\varphi_{i j}\left(t^{\prime}, \boldsymbol{z}^{\prime}, \tau^{\prime}, \tau, \mu\right)\right]} \\
& \times Q_{i j}\left(\boldsymbol{z}, \tau^{\prime}, \tau, \mu\right) \frac{A_{i j}\left(\boldsymbol{z}^{\prime}, t, \tau^{\prime}, \tau, \mu\right)}{B_{i j}\left(\boldsymbol{z}, t, \tau^{\prime}, \tau, \mu\right)}|t| d t^{\prime} d t d \tau d \mu .
\end{aligned}
$$

Using the stationary phase theorem [7] to approximate the $t^{\prime}$ and $\mu$ integrations, we obtain

$$
\begin{aligned}
& L_{i j}\left(\boldsymbol{z}, \boldsymbol{z}^{\prime}, \tau^{\prime}\right) \approx \\
& \int \mathrm{e}^{-\mathrm{i} \omega_{0} t\left[1-\left(\widehat{\left.\boldsymbol{\gamma}_{j}(\tau)-\mathbf{z}\right)} \cdot \dot{\boldsymbol{\gamma}}_{j}(\tau) / c_{0}\right]\left[S_{i j}\left(\tau^{\prime}, \tau, \boldsymbol{z}^{\prime}\right)-S_{i j}\left(\tau^{\prime}, \tau, \boldsymbol{z}\right)\right]\right.} \\
& \times Q_{i j}\left(\boldsymbol{z}, \tau^{\prime}, \tau\right) \frac{A_{i j}\left(\boldsymbol{z}^{\prime}, t, \tau^{\prime}, \tau\right)}{B_{i j}\left(\boldsymbol{z}, t, \tau^{\prime}, \tau\right)}|t| d t d \tau .
\end{aligned}
$$

Applying the method of stationary phase to the $t$ and $\tau$ integrals, we see that the main contribution to $L_{i j}\left(\boldsymbol{z}, \boldsymbol{z}^{\prime}, \tau^{\prime}\right)$ comes from those critical points of its phase that satisfy the conditions:

$$
S_{i j}\left(\tau^{\prime}, \tau, \boldsymbol{z}^{\prime}\right)=S_{i j}\left(\tau^{\prime}, \tau, \boldsymbol{z}\right)
$$

and

$$
\begin{array}{r}
a_{j}^{\sum}(\tau, \mathbf{z}) /\left[1-\left(\gamma_{j} \widehat{(\tau)-\mathbf{z}}\right) \cdot \dot{\gamma}_{j}(\tau) / c_{0}\right]= \\
a_{j}^{\sum}\left(\tau, \mathbf{z}^{\prime}\right) /\left[1-\left(\gamma_{j} \widehat{(\tau)-} \mathbf{z}^{\prime}\right) \cdot \dot{\gamma}_{j}(\tau) / c_{0}\right]
\end{array}
$$




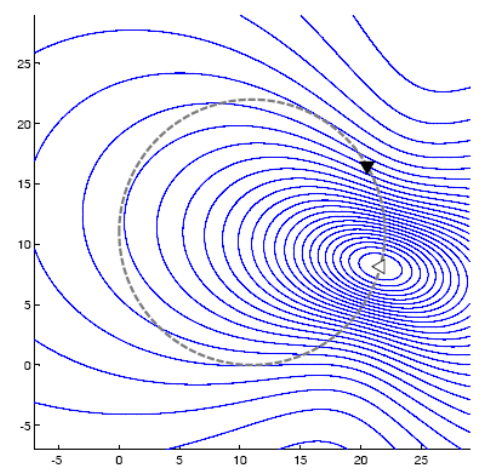

Fig. 3: Iso-Doppler-rate contours $\dot{F}_{j}(\tau, C)$ for the DSAH Doppler-rate $f_{1}(-\pi / 12, \mathbf{z})$. Two receivers are traversing a circular flight trajectory (dashed line) at the speed of $261 \mathrm{~m} / \mathrm{s}$ over a flat topography. The white and black triangles denote the positions of the two receivers .

where

$$
\begin{aligned}
& a_{j}^{\sum}(\tau, \mathbf{z})=\frac{1}{\left|\gamma_{j}(\tau)-\mathbf{z}\right|} \\
& \times\left[\dot{\gamma}_{j, \perp}(\tau, \mathbf{z}) \cdot \dot{\gamma}_{j}(\tau)\right]^{2}+\left(\widehat{\gamma_{j}(\tau)-} \mathbf{z}\right) \cdot \ddot{\gamma}_{j}(\tau) .
\end{aligned}
$$

Note that $\dot{\gamma}_{j, \perp}(\tau, \mathbf{z})$ is the projection of the receiver velocity $\dot{\gamma}_{j}(\tau)$ onto the plane whose normal direction is along $\gamma_{j}(\tau)-\mathbf{z}$ and $a_{j}^{\sum}(\tau, \mathbf{z})$ is the total relative radial acceleration of the $j$ th receiver in the direction of $\gamma_{j} \widehat{(\tau)-} \mathbf{z}$.

We define

$$
f_{j}(\tau, \mathbf{z}):=a_{j}^{\sum}(\tau, \mathbf{z}) /\left[1-\left(\widehat{\gamma_{j}} \widehat{(\tau)}-\mathbf{z}\right) \cdot \dot{\gamma}_{j}(\tau) / c_{0}\right]
$$

and refer to $f_{j}(\tau, \mathbf{z})$ as the DSAH Doppler-rate of the $j$ th receiver. We refer to the locus of points that is formed by the intersection of the surface topography and $\left\{\mathbf{z} \in \mathbb{R}^{3}\right.$ : $\left.f_{j}(\tau, \mathbf{z})=C\right\}$, for some constant $C$, as the DSAH isoDoppler-rate contour, and denote it by $\dot{F}_{j}(\tau, C)=\{\boldsymbol{z}$ : $\left.f_{j}(\tau, \boldsymbol{z})=C\right\}$. Figure 3 shows the DSAH iso-Dopplerrate contours with fixed $\tau$ for a circular flight trajectory and flat topography. The critical points $z$ of the phase of $L_{i j}\left(\boldsymbol{z}, \boldsymbol{z}^{\prime}, \tau^{\prime}\right)$ are those points lying on the intersection of the DSAH iso-Doppler curves $F_{i j}\left(\tau^{\prime}, \tau, \mu\right)$ and DSAH isoDoppler-rate curves $\dot{F}_{j}(\tau, C)$. We assume that the only critical point within the region of interest is $\boldsymbol{z}=\boldsymbol{z}^{\prime}$.

To determine the weight and the filter, we linearize $S_{i j}\left(\tau^{\prime}, \tau, \boldsymbol{z}^{\prime}\right)$ around $\boldsymbol{z}^{\prime}=\boldsymbol{z}$ to write

$$
\begin{array}{r}
L_{i j}\left(\boldsymbol{z}, \boldsymbol{z}^{\prime}, \tau^{\prime}\right)=\int \mathrm{e}^{-\mathrm{i} t \boldsymbol{\Xi}_{i j}\left(\tau^{\prime}, \tau, \boldsymbol{z}\right) \cdot\left(\boldsymbol{z}^{\prime}-\boldsymbol{z}\right)} Q_{i j}\left(\boldsymbol{z}, \tau^{\prime}, \tau\right) \\
\times \frac{A_{i j}\left(\boldsymbol{z}, t, \tau^{\prime}, \tau\right)}{B_{i j}\left(\boldsymbol{z}, t, \tau^{\prime}, \tau\right)}|t| d t d \tau
\end{array}
$$

where

$\boldsymbol{\Xi}_{i j}\left(\tau^{\prime}, \tau, \boldsymbol{z}\right)=\omega_{0}\left[1-\left(\widehat{\gamma_{j}(\tau)}-\mathbf{z}\right) \cdot \dot{\gamma}_{j}(\tau) / c_{0}\right] \nabla_{\boldsymbol{z}} S_{i j}\left(\tau^{\prime}, \tau, \boldsymbol{z}\right)$.
In (24) for each $\tau^{\prime}$ and $z$, we make the following change of variables:

$$
(t, \tau) \rightarrow \boldsymbol{\xi}_{i j}=t \boldsymbol{\Xi}_{i j}\left(\tau^{\prime}, \tau, \boldsymbol{z}\right)
$$

and we choose the weight and the filter as follows:

$$
Q_{i j}\left(\boldsymbol{z}, \tau^{\prime}, \tau\right)=\left[|t|,\left|\frac{\partial(t, \tau)}{\partial \boldsymbol{\xi}_{i j}}\right|\right]^{-1} \mid \operatorname{det}\left[\begin{array}{c}
\boldsymbol{\Xi}_{i j}\left(\tau^{\prime}, \tau, \boldsymbol{z}\right) \\
\partial_{\tau} \boldsymbol{\Xi}_{i j}\left(\tau^{\prime}, \tau, \boldsymbol{z}\right)
\end{array}\right] \text { (26) }
$$

and

$$
B_{i j}\left(\boldsymbol{z}, t, \tau^{\prime}, \tau, \mu\right)=\frac{\chi_{\Omega_{i j, \tau^{\prime}, \boldsymbol{z}}}\left(\boldsymbol{z}, t, \tau^{\prime}, \tau\right)}{A_{i j}\left(\boldsymbol{z}, t, \tau^{\prime}, \tau, \mu\right)}
$$

where $\chi_{\Omega_{i j, \tau^{\prime}, z}}$ is a smooth cut-off function equal to one in the interior of $\Omega_{i j, \tau^{\prime}, \boldsymbol{z}}$ and zero in the exterior of $\Omega_{i j, \tau^{\prime}, \boldsymbol{z}}$. We refer to $\Omega_{i j, \tau^{\prime}, \boldsymbol{z}}$ as the partial data collection manifold at $\left(\tau^{\prime}, \boldsymbol{z}\right)$ obtained by the $i$ th and $j$ th receivers for a fixed $\tau^{\prime}$ and refer to the union $\cup_{i j, \tau^{\prime}} \Omega_{i j, \tau^{\prime}, z}$ as the data collection manifold at $z$ and denote it by $\Omega_{z}$. This set determines many of the properties of the image.

Note that we choose the filter to compensate for the terms involving antenna beam patterns and geometric spreading functions and the weight to perform proper interpolation in the phase space going from $(t, \tau)$ to $\boldsymbol{\xi}_{i j}$ coordinates. These choices make the leading order term of $L_{i j}\left(\boldsymbol{z}, \boldsymbol{z}^{\prime}, \tau^{\prime}\right)$ in (24) to be the Dirac-delta function.

Substituting (25), (26) and (27) into (17), we obtain

$$
\begin{aligned}
& \tilde{R}_{\rho}(\boldsymbol{z})=\sum_{i j} \mathcal{K}_{i j}\left[\mathcal{F}_{i j}\left[R_{\rho}\right]\right](\boldsymbol{z}) \\
& \approx \sum_{i j} \int_{\Omega_{i j, \tau^{\prime}, \boldsymbol{z}}} \mathrm{e}^{-\mathrm{i} \boldsymbol{\xi}_{i j} \cdot\left(\boldsymbol{z}^{\prime}-\boldsymbol{z}\right)} R_{\rho}\left(\boldsymbol{z}^{\prime}\right) d \boldsymbol{z}^{\prime} d \boldsymbol{\xi}_{i j} d \tau^{\prime} .
\end{aligned}
$$

(28) shows that the image $\tilde{R}_{\rho}$ is a band-limited version of $R_{\rho}$ whose band-width is determined by the data collection manifold $\Omega_{\boldsymbol{z}}$, which describes the resolution of the reconstructed image $\tilde{R}_{\rho}$ at $z$. The larger the data collection manifold, the better the resolution of the image is.

Microlocal analysis of (28) tell us that an edge at point $z$ is visible if the direction $\boldsymbol{n}_{\boldsymbol{z}}$ normal to the edge is contained in $\Omega_{\boldsymbol{z}}$ [8]. Consequently, an edge at point $\boldsymbol{z}$ with $\boldsymbol{n}_{\boldsymbol{z}}$ normal to edge is visible if there exists $i, j, \tau^{\prime}, \tau$ such that $\boldsymbol{\xi}_{i j}$ is parallel to $\boldsymbol{n}_{\boldsymbol{z}}$. Furthermore, the band-width contribution of $\boldsymbol{\xi}_{i j}=t \boldsymbol{\Xi}_{i j}\left(\tau^{\prime}, \tau, \boldsymbol{z}\right)$ to a visible edge at $\boldsymbol{z}$ is given by $L_{\phi}\left|\boldsymbol{\Xi}_{i j}\left(\tau^{\prime}, \tau, \boldsymbol{z}\right)\right|$ where $L_{\phi}$ denotes the length of the support of $\phi(t)$. Thus, longer the support of $\phi(t)$, larger the magnitude of $\boldsymbol{\xi}_{i j}$ becomes, giving rise to sharper reconstructed edges perpendicular to $\boldsymbol{\xi}_{i j}, i, j=1, \ldots, N$. Additionally, higher the $\omega_{0}$, the frequency of the transmitted signal, larger the magnitude of $\boldsymbol{\xi}_{i j}$ becomes, contributing to higher image resolution.

(28) shows that irrespective of the choice of the weight and filter, the backprojection operator recovers the visible edges of the scene radiance at the right location for a given $i$ th and $j$ th receivers and a fixed $\tau^{\prime}$. With the choice of the weight and filter given in (26) and (27), respectively, the resulting image formation algorithm recovers the visible edges of the scene radiance not only at the right location and orientation, but also at the right strength. 


\section{NUMERICAL SimUlations}

We conducted numerical simulations to demonstrate the performance of our imaging method using a multiple-point-target model. We considered a scene of size $[0,22] \times[0,22] \mathrm{km}^{2}$ with flat topography. The scene was discretized by $128 \times 128$ pixels, where $[0,0,0] \mathrm{km}$ and $[22,22,0] \mathrm{km}$ correspond to the pixels $(1,1)$ and $(128,128)$, respectively.

In all the numerical experiments, we used two airborne receivers and a single, stationary transmitter operating either cooperatively or non-cooperatively. We assumed that both the receiver and transmitter antennas were isotropic. We assumed that the transmitter was located at $\mathbf{y}_{0}=(0,0,6.5) \mathrm{km}$ and the receivers were traversing the circular trajectory given by

$$
\gamma_{C}(s)=(11+11 \cos (s), 11+11 \sin (s), 6.5) \mathrm{km} .
$$

Let $\gamma_{1}(s)$ and $\gamma_{1}(s)$ denote the trajectories of the two receivers. We set $\gamma_{1}(s)=\gamma_{C}(s)$ and $\gamma_{2}(s)=\gamma_{1}\left(s-\frac{\pi}{6}\right)$. Note that the variable $s$ in $\gamma_{C}$ is equal to $\frac{V}{R} t$ where $V$ is the speed of the receiver, and $R$ is the radius of the circular trajectory. We set the speed of the two receivers to $261 \mathrm{~m} / \mathrm{s}$. We chose the sampling rate of $\tau$ to be $1.9335 \mathrm{~Hz}$ so as to uniformly sample the circular trajectory with 512 points.

For all the numerical experiments, we used (1) to generate the data and chose the windowing function $\phi$ in (3) to be a Hanning function. We performed image reconstruction for each $\tau^{\prime}$ and coherently superimposed the reconstructed images obtained over a range of $\tau^{\prime}$.

In accordance with the incoherent field approximation, we used the following multiple-point-target model for the scene reflectivity

$$
\rho(\boldsymbol{z})=\sum_{l=1}^{L} g_{l} \delta\left(\boldsymbol{z}-\boldsymbol{z}_{l}\right)
$$

where $g_{l}, l=1, \cdots, L$ are independent Gaussian random variables with mean $\mu_{l}$ and variance $\sigma_{l}^{2}$. The corresponding scene radiance is given by

$$
R_{\rho}(\boldsymbol{z})=E\left[\rho(\boldsymbol{z}) \rho^{*}(\boldsymbol{z})\right]=\sum_{l}\left(\mu_{l}^{2}+\sigma_{l}^{2}\right) \sigma\left(\boldsymbol{z}-\boldsymbol{z}_{l}\right) .
$$

In our simulations, we considered a deterministic reflectivity and set $\sigma_{l}^{2}=1$. We used $L=9$ and approximated the Dirac-delta functions in (31) by square target reflectors of size $344 \times 344 \mathrm{~m}^{2}$, each having a unit reflectivity, i.e., $\mu_{l}=1, l=$ $1, \cdots, 9$. The Figs.4(a) and 4(b) show the scene with targets, receiver trajectories and the transmitter antenna location.

\section{A. Cooperative Transmitters -}

For the case of a cooperative transmitter, the reconstructed image is shown in Fig.5. The two images corresponding to the fixed $\tau^{\prime}$ values are shown in Fig.6(a) and Fig.6(b). As expected, due to a smaller data collection manifold, the resolution of these images is poorer than that of the image obtained by superposition of all the images reconstructed for a range of $\tau^{\prime}$ values, $\tau^{\prime} \in[0,248.258] \mathrm{s}$, as shown in Fig.5. We observe that some of the targets do not appear as sharp as the ones in Fig.5.
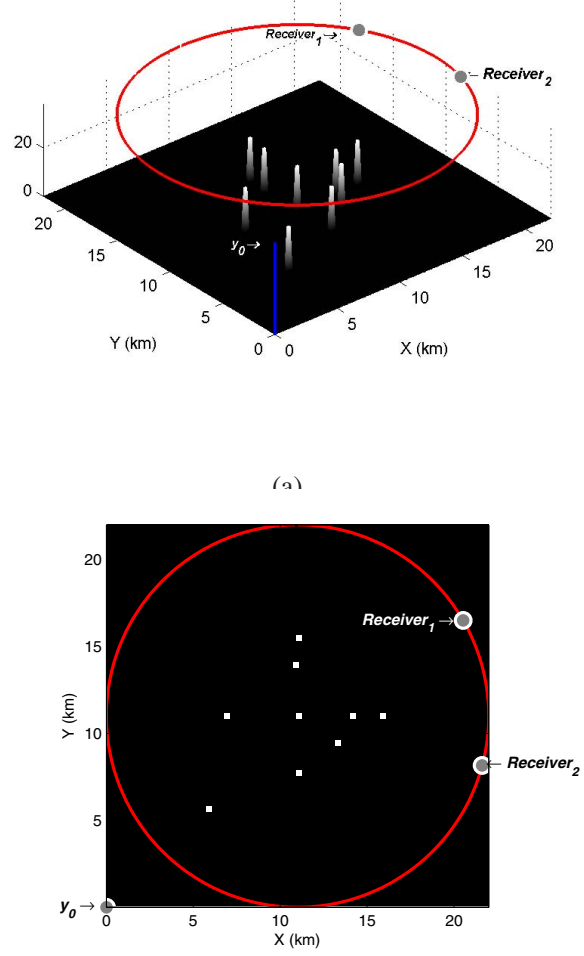

(b)

Fig. 4: (a) 3-D and (b) 2-D views of the scene with multiple point targets, illuminated by a single transmitter located at $\mathbf{y}_{0}=(0,0,6.5) \mathrm{km}$ and the circular receiver trajectory $\gamma_{C}(s)=(11+11 \cos (s), 11+11 \sin (s), 6.5) \mathrm{km}$, as shown by the red solid line. At a certain time instant, two receivers are located at the positions shown in the figure.

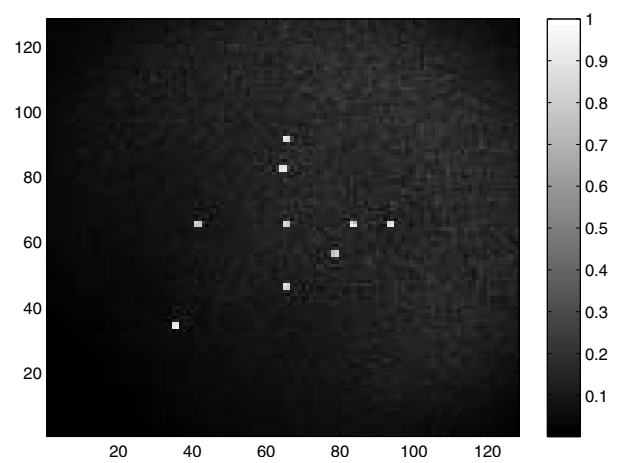

Fig. 5: The reconstructed image for multiple point targets with $16 \tau^{\prime}$ values uniformly spaced in $[0,248.258] \mathrm{s}$ using a single cooperative transmitter located at $\mathbf{y}_{0}$ and two receivers traversing the circular flight trajectories $\gamma_{1}(s)$ and $\gamma_{2}(s)$ as shown in Fig.4.

\section{B. Non-cooperative Transmitters -}

Fig.7 shows the reconstructed image for multiple point targets using a non-cooperative transmitter. Since the location 

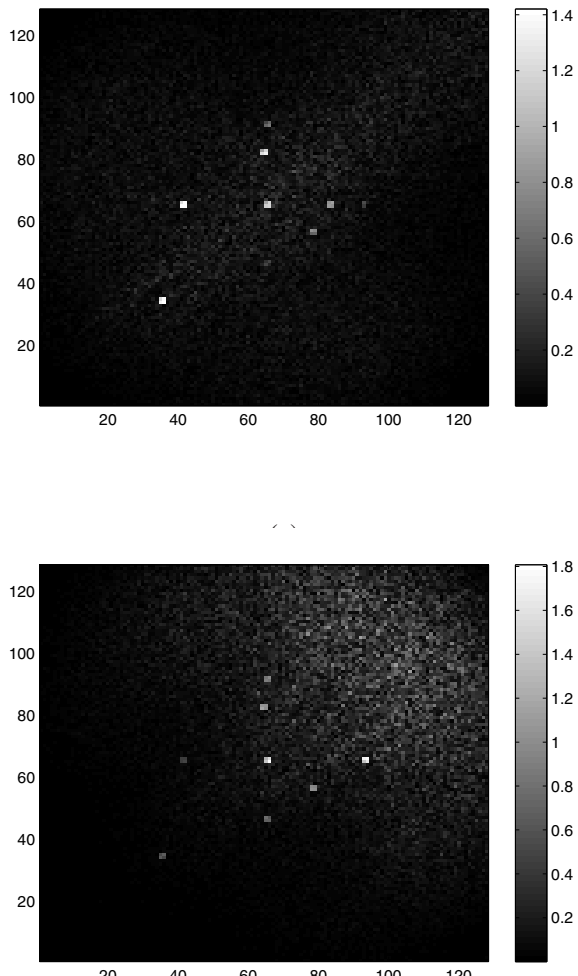

(b)

Fig. 6: The images reconstructed for multiple point targets using the vectors in the partial data collection manifolds (a) $\Omega_{12, \tau^{\prime}=33.1011 \mathrm{~s}}$ (corresponding to $s=\pi / 4$ ), and (b) $\Omega_{12, \tau^{\prime}=165.5054 \mathrm{~s}}$ (corresponding to $s=1.25 \pi$ ), with a single cooperative transmitter located at $\mathbf{y}_{0}$ and two receivers traversing circular flight trajectories $\gamma_{1}(s)$ and $\gamma_{2}(s)$ as shown in Fig.4.

of the transmitter was assumed to be unknown, the received signal was not compensated for the transmitter related geometric spreading factors. As a result, the scatterers closer to the transmitter appears brighter in the reconstructed image than those that are further away from the transmitter.

\section{CONCLUSION}

In this paper, we developed a novel image formation method for passive SAR that uses transmitters of opportunity with fixed-frequency waveforms and receivers traversing arbitrary flight trajectories. The method is based on the windowed, filtered-scaled-and-translated correlation of the received signals at different (or the same) receiver and weightedbackprojection of the resulting correlated signal. Such preprocessing of the received signal removes the transmitter related terms from the phase of the resulting forward model allowing us to perform backprojection without having the knowledge of transmitter locations.

The analysis of the point spread function of the imaging operator shows that the weighted-backprojection algorithm puts

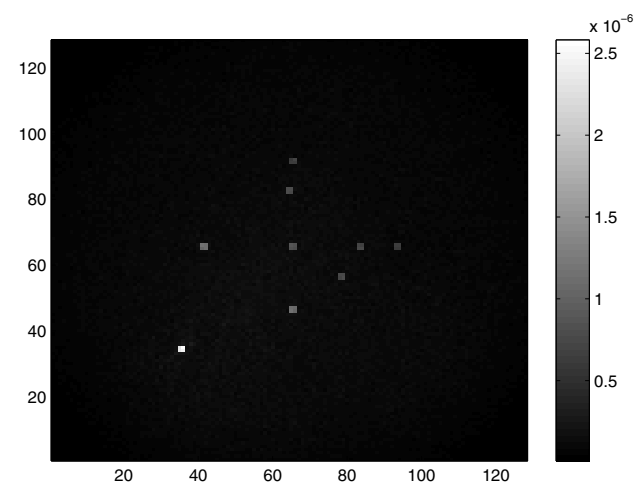

Fig. 7: The reconstructed image for multiple point targets with $16 \tau^{\prime}$ values uniformly sampled in $[0,248.258] \mathrm{s}$ using a single non-cooperative transmitter located at $\mathbf{y}_{0}$ and two receivers traversing the circular flight trajectories $\gamma_{1}(s)$ and $\gamma_{2}(s)$ as shown in Fig.4.

the visible edges of the scene radiance at the correct location, and under appropriate conditions, with correct strength. The numerical simulations demonstrate the performance of the reconstruction algorithm.

We described our imaging algorithm for a single, stationary transmitter. However, the method can be easily extended to multiple mobile or stationary transmitters by simply integrating the received signal model over all transmitter locations similar to our work in [4]. The method can be extended to include the case where the received data is contaminated with additive noise following the framework introduced in our prior work [9].

Although we presented our approach specifically for passive synthetic aperture radar, our method is also applicable to other passive imaging problems, such as passive geophysical or acoustic imaging.

\section{REFERENCES}

[1] H. D. Griffiths and C. J. Baker, "Passive coherent location radar systems. part 1: Performance prediction," IEE Proc.-Radar Sonar Navig., vol. 152, no. 3, June 2005.

[2] D. Poullin, "Passive detection using digital broadcasters (DAB, DVB) with COFDM modulation," IEE Proc.-Radar Sonar Navig., vol. 152, no. 3, pp. 143-152, June 2005.

[3] R. Pollard, "The role of passive radar sensors for air traffic control," in The Institution of Engineering and Technology Seminar on the Future of Civil Radar, 2006.

[4] C.E. Yarman and B. Yazıcı, "Synthetic aperture hitchhiker imaging," IEEE Transactions on Imaging Processing, vol. 17, no. 11, pp. 21562173, Nov. 2008.

[5] C.J. Nolan and M. Cheney, "Synthetic aperture inversion for arbitrary flight paths and non-flat topography," IEEE Transactions on Image Processing, vol. 12, pp. 1035-1043, 2003.

[6] H.H. Barrett and K.J. Myers, Foundations of image science, WileyInterscience, Hoboken, NJ, 2004.

[7] N. Bleistein and R.A. Handelsman, Asymptotic Expansions of Integrals, Dover, New York, 1986.

[8] C. J. Nolan and M. Cheney, "Synthetic aperture inversion," Inverse Problems, vol. 18, pp. 221-236, 2002.

[9] B. Yazici, M. Cheney, and C.E. Yarman, "Synthetic-aperture inversion in the presence of noise and clutter," Inverse Problems, vol. 22, pp. 1705-1729, 2006. 